\title{
Comparaciones múltiples no paramétricas en la evaluación sensorial de la apariencia y sabor de tres marcas de cerveza comercial
}

\section{Nonparametric multiple comparisons in sensory evaluation of appearance and flavor of three brands of commercial beer}

\author{
Carlos Minchón ${ }^{1}$, Erika Mío y Kathy Córdova ${ }^{3}$
}

\author{
${ }^{1}$ Universidad Nacional de Trujillo, cminchonm@gmail.com \\ 2 Universidad Nacional de Trujillo, firstkiss17@hotmail.com \\ ${ }^{3}$ Universidad Nacional de Trujillo, kacese_4@hotmail.com
}

\section{RESUMEN}

La evaluación sensorial de los alimentos constituye una de las más importantes herramientas en la industria alimentaria. Las técnicas de evaluación sensorial aplicadas a quesos, embutidos, miel, agua, aceite de oliva y vino, también pueden ser empleadas en procesos de degustación con consumidores potenciales para conocer la percepción sobre las características organolécticas de productos en elaboración y productos comerciales, generándose la necesidad de difundir la aplicación de métodos estadísticos no paramétricos. $\mathrm{El}$ propósito del presente trabajo es determinar la marca de cerveza comercial con mejor apariencia y sabor empleando las pruebas no paramétricas de comparaciones múltiples. Las cervezas Pilsen Trujillo, Cristal y Brahma, marcas con importante aceptación en el mercado trujillano, fueron evaluadas sensorialmente en apariencia y sabor por 60 consumidores potenciales, usando escalas hedónicas de 5 puntos. Se aplicó la prueba de Friedman y pruebas de comparaciones múltiples, incluyendo pruebas considerando la cerveza Pilsen Trujillo. Los datos fueron procesados en IBM SPSS Statistics 19 y en EXCEL. La prueba de Friedman estableció diferencia tanto en la apariencia $(p=0.000<0.01)$ como en el sabor $(p=0.000<0.01)$ entre marcas, destacando Pilsen Trujillo en apariencia y Cristal en el sabor. Las pruebas de comparaciones múltiples evidenciaron similitudes de Pilsen Trujillo con Cristal en la apariencia ( $p>0.05)$ y con Brahma en el sabor ( $p>0.05)$, pero ambas por debajo de Cristal en cuanto al sabor. Se concluye que la cerveza Cristal es la mejor en sabor y similar en apariencia Pilsen Trujillo, pero superior a Brahma en ambos atributos. Además, la facilidad de aplicación de los métodos no paramétricos para comparaciones múltiples por rangos permiten extender su uso en la evaluación sensorial de productos tanto en proceso de elaboración como comerciales.

Descriptores: evaluación sensorial, comparaciones múltiples, no paramétrico, cerveza.

\section{ABSTRACT}

The sensory evaluation of food is an important tool in the food industry. Sensory evaluation techniques applied to cheeses, cured meats, honey, water, olive oil and wine, can also be used in potential consumers tasting process to determine the perception of the organoleptic characteristics in elaboration of products and commercial products, generating the need to spread the application of nonparametric statistical methods. The purpose of this study is to determine the commercial beer brand with better appearance and flavor using nonparametric multiple comparisons. Trujillo Pilsen, Cristal and Brahma, brands with important acceptance in Trujillo market, were evaluated in appearance and flavor sensory for 60 potential customers using a 5-point hedonic scale. Was applied the Friedman test and multiple comparison tests and tests with a control, the control was Pilsen Trujillo beer. The data were processed by IBM SPSS 19 and Excel. Friedman test showed difference both in appearance $(p=0.000<0.01)$ and flavor $(p=0.000<0.01)$ between brands, Pilsen Trujillo in appearance and Cristal in flavor. Multiple comparison tests showed similarities between Cristal and Trujillo Pilsen beers in appearance ( $p>0.05)$ and with Brahma beer in flavor ( $p>0.05)$, both inferiors to Cristal in flavor. Was conclude that Cristal beer is the best in flavor and similar in appearance with Pilsen Trujillo, but 
higher than Brahma in both attributes. Furthermore, the ease application of nonparametric methods for multiple comparisons by ranks, let us to extend its use in sensory evaluation in elaboration of products and commercial products.

Keywords: sensory evaluation, multiple comparisons, nonparametric, beer.

\section{INTRODUCCIÓN}

La evaluación sensorial de los alimentos constituye una de las más importantes herramientas en la industria alimentaria [1]. A nivel de consumidor se aplica para comprender la importancia de las propiedades de aceptación-rechazo, preferencia y nivel de agrado, en relación con los atributos del mismo producto [2]. El nivel de agrado por los alimentos en respuesta a cómo cumple con las expectativas se evalúa con escalas hedónicas a partir de la apreciación de agrado o desagrado de potenciales consumidores [1].

Las evaluaciones sensoriales en las que se ha empleado una escala hedónica para realizar análisis sensorial de los productos que se investiga, usualmente se recomienda el uso de la prueba de Friedman para realizar el análisis estadístico [2,3], prueba no paramétrica para comparar más de dos tratamientos valorados en escala ordinal [4]. Sin embargo, no siempre se hace el uso de la prueba de Friedman o de las pruebas de comparaciones múltiples, o se hace uso indebido de otras pruebas.

Evidencias al respecto, se tienen en la evaluación sensorial de la apariencia, aroma, consistencia y sabor de tres bebidas saborizadas obtenidas en base a arroz y soya [5], o el caso de la aceptabilidad de cuatro bebida alcohólicas fermentadas producidas en base a camu camu [6], en los que se empleó erróneamente el análisis de varianza (ANVA) y la prueba de Tuckey en comparaciones múltiples. Sin embargo, la posibilidad de un uso adecuado de las pruebas de comparaciones múltiples, además de la prueba de Friedman, podría darse en pocos trabajos, como en el caso de la producción de una pasta de aceituna en el que se aplicó la prueba de diferencia mínima significativa de Fisher para rangos en la comparación por pares del color de las pastas en diferentes condiciones de elaboración [7] o en el caso de la preferencia de tres bebidas lácteas fermentadas con diferentes concentraciones de suero de queso [8].

La prueba de Friedman fue utilizada para evaluar la satisfacción organoléctica de tres marcas de hotdog clásico [9] y en la evaluación de la apariencia y color de tres marcas de cerveza [10]. Sin embargo, dada la poca difusión de otras pruebas se recurrió a la prueba de Wilcoxon para establecer las "mejores" entre las marcas en evaluación en una comparación por pares. Sin embargo, la prueba de Wilcoxon se recomienda para comparaciones de dos tratamientos planeada antes de la recolección de datos [11].

La cerveza es un producto indispensable, sin embargo en el Perú se consumen anualmente alrededor de 12 millones de hectolitros, alcanzando el consumo percápita entre 41 a 42 litros por persona. Aún cuando el consumo de cerveza alcanza un nivel importante en el país, las empresas ya hace algún tiempo han iniciado una dura batalla para posicionarse en el mercado empleando una serie de estrategias tendientes a mantener a sus consumidores o atrayendo a consumidores de las marcas competidoras, y tratando que las nuevas marcas fracasen en su intento por posicionarse en su mercado.

La cerveza no tiene como fin primario la alimentación de la población, pero debido a que contiene una cantidad importante de carbohidratos, vitaminas y proteínas, hasta se considera que un consumo "muy moderado es "benéfico" para la salud. Las técnicas de evaluación sensorial aplicadas a quesos, embutidos y miel, así como al agua, aceite de oliva y los vinos [1], también puede ser ampliado al estudio sensorial de la cerveza. Sin embargo, el análisis de la data generada en la degustación de los productos no será adecuadamente realizado si no se conoce los métodos estadísticos no paramétricos específicos para este proceso.

El propósito del presente trabajo es determinar la marca de cerveza comercial con mejor apariencia y sabor empleando las pruebas no paramétricas de comparaciones múltiples.

\section{METODOLOGÍA}

En presente trabajo se realiza la evaluación sensorial de la apariencia y sabor de tres marcas de cerveza en potenciales consumidores varones, con edades entre 18727 años [10]. Las marcas de cerveza evaluadas fueron Pilsen Trujillo (marca local), Cristal y Brahma, marcas de mayor consumo [12].

Se empleó un total de 60 potenciales consumidores, quienes realizaron la cata de las cervezas en un local acondicionado, empleando para la evaluación 
ECIPERÚ

una escala hedónica de cinco puntos (1: me disgusta mucho, 2: no me gusta, 3: no me gusta ni me disgusta, 4: me gusta, 5: me gusta mucho).

Las muestras de cerveza fueron presentadas a los consumidores en vasos de vidrio de $63 / 4$ onzas, con paredes lisas, sin relieve ni decoración añadida, muy limpios. Las muestras, previamente codificadas, fueron presentadas ordenadamente a cada panelista para su degustación (de izquierda a derecha), con opción a reprobar (en el caso del sabor, tenían que proceder a enjuagar con agua). Cada panelista se constituye en un bloque, en términos experimentales.

Las muestras fueron colocadas al azar, de manera que cada cerveza apareciera el mismo número de en cada posición.

El análisis estadístico de los datos sensoriales se realizó empleando IBM SPSS Statistics 19 para realizar la prueba de Friedman, reportándose los rangos promedio para cada marca de cerveza. En esta prueba, la hipótesis de interés es que no hay efecto de la marca de cerveza, es decir se somete a prueba $[4,12]$ :

$$
\begin{aligned}
& H_{o}: \tau_{1}=\tau_{2}=\cdots=\tau_{s}
\end{aligned}
$$

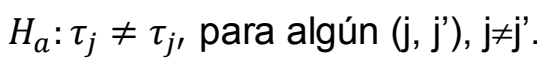

Adicionalmente, se recomiendan en este caso las siguientes pruebas basadas en los rangos de Friedman.

\section{COMPARACIONES MÚLTIPLES ENTRE TODAS LAS CERVEZAS}

Sean las hipótesis

$$
H_{o}: \tau_{j_{1}}=\tau_{j_{2}} \quad H_{a}: \tau_{j_{1}} \neq \tau_{j_{2}}
$$

Para cada par de tratamientos (cervezas) en comparación $\left(\mathrm{j}_{1}, \mathrm{j}_{2}\right), \mathrm{j}_{1}>\mathrm{j}_{2}$, considere el estadístico:

$$
Z_{j_{1}, j_{2}}=\frac{\left|r_{j_{1}}-r_{j_{2}}\right|}{\sqrt{s(s+1) / 12 r}}
$$

Donde $s=3$ es el número de cervezas y $r=60$ el número de consumidores. La hipótesis nula es rechazada si $Z_{j_{1}, j_{2}}>\mathrm{QP}_{\alpha}(\mathrm{s})[2,12]$. Los valores $\mathrm{QP}_{\alpha}(\mathrm{s})$ constituyen las amplitudes para rangos promedios, correspondientes a los $s$ tratamientos y error tipo I igual a $\alpha$, los cuales se encuentran tabulados.

\section{COMPARACIÓN DE UNA CERVEZA FRENTE A LAS OTRAS MARCAS}

Sea j=1 el subíndice de la cerveza control, la cerveza Pilsen Trujillo (marca local), la cual puede ser planteada como:

$$
H_{o}: \tau_{1}=\tau_{j} \quad H_{a}: \tau_{1} \neq \tau_{j}, j \neq 1
$$

El estadístico de prueba para la comparación múltiple de la marca 1 con las (s-1) marcas restantes es:

$$
Z_{1 j}=\frac{\left|r_{1}-r_{j}\right|}{\sqrt{s(s+1) / 6 r}}, j=2,3, \ldots, s
$$

La hipótesis nula se rechaza si $Z_{1 j}>\mathrm{QC}_{\alpha}(\mathrm{s}-1)$ [12].

\section{RESULTADOS Y DISCUSIÓN}

Las preferencias de las cervezas Pilsen Trujillo, Cristal y Brahma evaluadas sensorialmente en cuanto a apariencia y sabor por 60 consumidores potencias, usando escalas hedónicas con puntajes de 1 a 5 , se sintetizan en los rangos promedios determinados a través de la prueba de Friedman, los cuales se muestran en la tabla 1. El programa IBM SPSS Statistics 19, considera los posibles empates en los tratamientos comparados, corrigiendo el estadístico de prueba, según como se indica en la literatura.

Tabla 1. Prueba de Friedman de la apariencia y sabor de tres marcas de cerveza.

\begin{tabular}{lrr}
\hline \multirow{2}{*}{ Marca de } & \multicolumn{2}{c}{ Rangos promedios } \\
\cline { 2 - 3 } cerveza & Apariencia & \multicolumn{1}{c}{ Sabor } \\
\hline Pilsen Trujillo & 2.44 & 1.66 \\
Cristal & 2.06 & 2.58 \\
Brahma & 1.50 & 1.76 \\
\hline Chi-cuadrado & 30.178 & 30.584 \\
$\mathrm{P}$ & 0.000 & 0.000 \\
\hline
\end{tabular}

La cerveza Pilsen Trujillo alcanzó el mayor rango en apariencia (2.44) pero el más pequeño en sabor (1.66). La cerveza Cristal es la que muestra el mejor rango en sabor (2.58) y en apariencia ocupa el segundo lugar (2.06). La cerveza Brahma, presenta el menor rango en apariencia (1.50), pero el segundo en sabor (1.76). Los rangos presentados son los proporcionados en una de las referencias [10], los cuales se muestran gráficamente en la 
figura 1, sobresaliendo los ejes de la cerveza Pilsen Trujillo en apariencia y la cerveza Cristal en sabor.

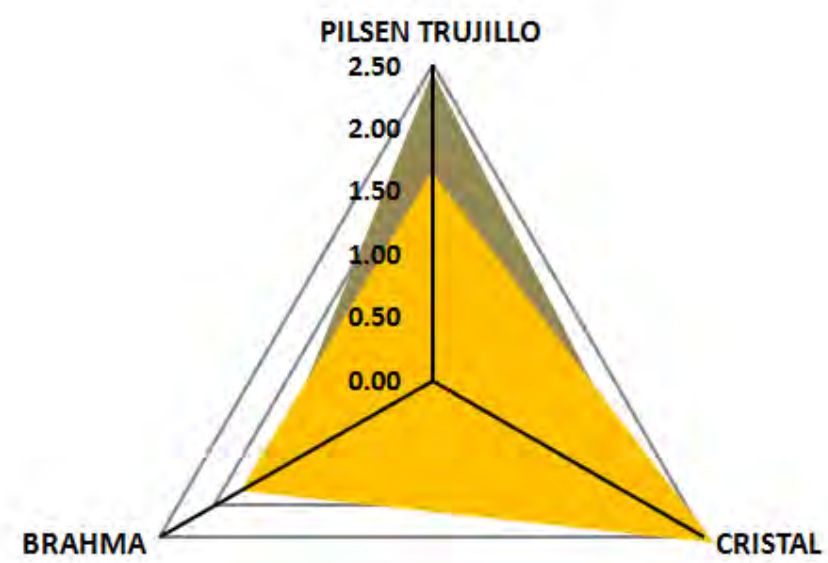

Apariencia $=$ Sabor

Figura 1. Rangos promedios en apariencia y sabor de las cervezas Pilsen Trujillo, Cristal y Brahma.

Es de esperarse que las marcas que más gustan a los consumidores tengan los mejores indicadores en un estudio de mercado. En cuanto a la cerveza, la Pilsen Trujillo fue considerada el 2010 en la Ciudad de Trujillo como la mayor marca habitual de consumo el 2010 (67\%), Cristal en tercer lugar $(11 \%)$ y Brahma en quinto lugar $(9 \%)$ [12], asumimos que las diferencias se debe a que en este trabajo la población objetivo fueron hombres y mujeres de 18 a 70 años.

Las diferencias mostradas por las marcas de cerveza Pilsen Trujillo, Cristal y Brahma, alcanzaron significación estadística, tanto en apariencia $(p=0.000<0.05)$ como en sabor $(p=0.000<0.05)$. Sin embargo, un análisis más específico de las marcas de cervezas realizadas por pares con el fin de determinar la marca con mejor apariencia y sabor según la evaluación sensorial de los consumidores se presenta en la tabla 2, las cuales corresponden a las pruebas de hipótesis (2) indicadas en la metodología.

Tabla 2. Comparaciones múltiples de la apariencia y sabor de tres marcas de cerveza.

\begin{tabular}{lcccc}
\hline \multicolumn{1}{c}{ Marcas } & \multicolumn{2}{c}{ Apariencia } & \multicolumn{2}{c}{ Sabor } \\
\multicolumn{1}{c}{ comparadas } & $/ \mathrm{DR} / /^{1}$ & $\mathrm{Z}^{2}$ & \multicolumn{1}{c}{$/ \mathrm{DR} /{ }^{1}$} & $\mathrm{Z}^{2}$ \\
\hline Trujillo/Cristal & 0.38 & 2.943 & 0.92 & 7.126 \\
Trujillo/Brahma & 0.94 & 7.281 & 0.10 & 0.775 \\
Cristal/Brahma & 0.56 & 4.338 & 0.82 & 6.352 \\
\hline
\end{tabular}

${ }^{1} / \mathrm{DR} /$ : Valor absoluto de la diferencia de rangos
${ }^{2} \mathrm{QP} 5 \%(3)=3.314$ y $\mathrm{QP} 1 \%(3)=4.120$.

Las pruebas mostradas en la tabla 2, revelan que Pilsen Trujillo es la cerveza de mejor apariencia para la muestra, pero su diferencia con la Cristal no es estadísticamente significativa $(Z=2.943<3.14)$, pero si con la apariencia de la Brahma $(Z=7.281>4.120)$. Por su parte, Cristal es la cerveza que para la muestra tenía el mejor sabor, alcanzando nivel estadísticamente significativo, incluso al $1 \%$, con el sabor tanto de la cerveza Pilsen Trujillo $(Z=7.126>4.120)$ como de la cerveza Brahma $(Z=6.352>4.120)$.

La aplicación de la prueba de Wilcoxon a la apariencia proporciona diferencia estadísticamente significativa entre todos los pares de marcas en cuanto a la apariencia, incluso entre Pilsen Trujillo y Cristal, pero coincidencia en las comparaciones del sabor [10].

En la evaluación sensorial realizada, Pilsen Trujillo destaca por su presencia y Cristal por su sabor, no encontrándose una neta ventaja de una de las marcas, pero si una tendencia de la cerveza Cristal a ser la más aceptada entre las cerveza en evaluación, de acuerdo a los atributos apariencia y sabor. La apariencia es el aspecto exterior resultante de apreciar su color, forma, tamaño, estado y características de su superficie; y el sabor es la combinación del olor, aroma, gusto y textura [3]. En la cerveza, la graduación alcohólica, el amargor, el cuerpo y la temperatura determinan el sabor.

La ausencia de una marca percibida sensorialmente como la mejor en todos atributos se explica porque en la evaluación sensorial no es posible elegir una marca por uno de los atributos, las pruebas sensoriales no permiten discriminar entre un atributo u otro, ya que por lo general las sensaciones experimentadas al ingerir una bebida no es producido por un solo sentido, sino que en ella se entremezclan distintos estímulos actuando como respuesta a la estimulación compleja [1]. La preferencia por una de las marcas se hace más complicada en la medida que se seleccione mayor número de atributos.

Una alternativa al estadístico presentado para la prueba (2), es la diferencia mínima significativa (DMS) para comparación múltiple de la suma de rangos, en la cual se compara la diferencia de la suma de rangos de cada par de tratamientos con el valor DMS[2], en cual es dado por:

$$
D M S=Q P_{\alpha}(s) \sqrt{\frac{r s(s+1)}{12}}
$$


Este procedimiento aparentemente fue aplicado en la comparación de las bebidas lácteas fermentadas con diferentes concentraciones de queso [8], por un lado por lo expresado en su análisis estadístico y por el valor crítico de 21 que reporta al $5 \%$, el cual coincidiría con el DMS correspondiente a $s=3$ tratamientos y $r=40$ evaluadores. Más no se puede aseverar completamente debido a que no se reporta en número real de evaluadores.

Finalmente, como un modo de evaluar el regionalismo de la población, se consideró a la cerveza Pilsen Trujillo como la marca control, para compararla con las cervezas Cristal y Brahma. Los resultados se muestran en la tabla 3 , donde las hipótesis en prueban son las del tipo 3 , presentadas en la metodología.

Tabla 3. Comparaciones en apariencia y sabor de la cerveza Pilsen Trujillo con las cervezas Cristal y Brahma.

\begin{tabular}{lcccc}
\hline \multicolumn{1}{c}{ Marcas } & \multicolumn{2}{c}{ Apariencia } & \multicolumn{2}{c}{ Sabor } \\
\multicolumn{1}{c}{ comparadas } & $/ \mathrm{DR} /{ }^{1}$ & $\mathrm{Z}^{2}$ & $\mathrm{IDR} /{ }^{1}$ & $\mathrm{Z}^{2}$ \\
\hline Trujillo/Cristal & 0.38 & 2.081 & 0.92 & 5.039 \\
Trujillo/Brahma & 0.94 & 5.149 & 0.10 & 0.548 \\
\hline ThR/: Valor absoluto de la diferencia de rangos \\
${ }^{\text {2 }}$ QC5\%(2)=2.21.
\end{tabular}

Los hallazgos muestran que la cerveza Pilsen Trujillo no se diferencia en apariencia de la cerveza Cristal $(Z=2.081<2.21)$, pero la diferencia con la cerveza Brahma es real $(Z=5.149>2.21)$. Pero en sabor, la diferencia con la cerveza Cristal $(Z=5.039>2.21)$, pone en desventaja a la cerveza Pilsen Trujillo, y no se diferencia de la Brahma $(Z=0.548<2.21)$.

En realidad, los resultados mostrados en la tabla 3 corroboran los de la tabla 2. Aún a pesar de esto, siempre que haya un tratamiento control la prueba de comparaciones múltiples se debe complementar con la prueba que compara los otros tratamientos con el control (si lo hay), debido a que si bien los estadísticos en las pruebas de hipótesis (2) y (3) tienen entre sí un factor de proporcionalidad, dado por $\sqrt{2}$, los puntos críticos en las respectivas tablas no guardan la misma proporcionalidad.

Una alternativa a esta prueba lo constituye la prueba de diferencia mínima significativa de Fisher para rangos, cuyas siglas en inglés son LSRD [13], la cual está dada por:

$$
L S R D=t_{\alpha / 2 \infty} \sqrt{r S(s+1) / 6}
$$

Donde, $t_{\alpha / 2 \infty}$ es tomado de la distribución $t$ de Student, aproximada la normal. El valor 6 en el divisor de la raíz cuadrada corresponde justamente a la comparación múltiple por pares. Sin embargo, puede apreciarse que el valor $t_{\alpha / 2 \infty}$ es fijo, no considera el número de tratamientos en comparación, lo cual pareciera ser una limitación.

En la comparación por pares de las tres bebidas lácteas fermentadas con diferentes concentraciones de suero de queso según se indica en metodología de ha aplicado esta prueba [8]. De ser cierto lo expresado por los autores, se realizó una aplicación inadecuada de la prueba, debido a que en ninguna parte se revela que haya un grupo control. Sin embargo, al igual que el anterior estudio, no se reporta los rangos obtenidos por cada producto en la prueba de Friedman que permita verificar los resultados.

Las pruebas detalladas en el presente trabajo son fácilmente aplicables, permitieron establecer las diferencias entre las marcas de cerveza, pero en contraposición se ha mostrado que no han sido muy difundidas, incluso en los trabajos realizados en nuestra Universidad, en las que se recurrió a la prueba de Wilcoxon $[9,10]$.

\section{CONCLUSIONES}

La cerveza Cristal es la mejor en sabor y similar en apariencia Pilsen Trujillo, y superior a Brahma en ambos atributos, según la evaluación sensorial realizada por los potenciales consumidores.

Además, la facilidad de aplicación de los métodos no paramétricos para comparaciones múltiples por rangos permiten extender su uso en la evaluación sensorial de productos tanto en proceso de elaboración como comerciales.

\section{AGRADECIMIENTOS}

Agradecimiento especial a la Escuela de Estadística de la Universidad Nacional de Trujillo, por hacer posible la presencia en el ECl2011i.

\section{REFERENCIAS}

[1] J. Sancho, E. Bota, J.J. de Castro. Introducción al análisis sensorial de los alimentos. Universidad de Barcelona, 1999.

E. J. Espinoza. Evaluación sensorial de los alimentos, Universidad Nacional Jorge Basadre Grohmann, 2003.

[3] M. Ureña, M. D’Orrigo, O. Girón. Evaluación 
Sensorial de los Alimentos, Universidad Nacional Agraria La Molina, 1999.

[4] N. Sprent, M.C. Smeeton. Applied nonparametric statistical methods, third edition, Chapman \& Hall/CRC, 2001.

[5] M. Soares et al. Bebidas saborizadas obtidas de extratos de quirera de arroz, de arrpz integral e de soja, Cienc. Agrotec, 2010, 34(2): 407-413.

[6] R.N. Maeda, J.S. Andrade. Aproveitamento do camu-camu (Myrciaria dubia) para produção de bebida alcohólica fermentada, Acta Amazónica, 33(3): 489-496.

[7] M. L. Escudero, A. J. Meléndez, F. J. Heredia, I. M. Vicario. Optimization of olive-fruit paste production using a methodological proposal based on a sensory and objective color analysis, Grasas y aceites, 2009, 60(4): 396404.

[8] V. M. de Oliveira, M. A. Slobosa, M. Q. de Freitas, R.M. Franco. Avaliação sensorial de bebida láctea fermentada com diferentes concentrações de soro de queijo, enriquecida com ferro, R. Bras. Ci. Vet., 2006, 13(2): 67.70, en

[9] E. J. García. Comparación de la satisfacción organoléctica en tres marcas de hot dog clásico de venta en mercados de Trujillo, tesis, Universidad Nacional de Trujillo, 2011.

[10] E. P. Mío. Evaluación hedónica de tres marcas de cerveza para consumidores trujillanos, diciembre 2010, tesis, Universidad Nacional de Trujillo, 2011.

[11] H. Toutenburg. Statistical Analysis of Designed Experiments, second edition, Springer, 2002.

[12] Investiga. Top of mind, Marcas recordadas y preferidas por los trujillanos. Universidad Privada Antenor Orrego, 2010, en http://www.upao.edu.pe/upload/recursos/investi ga/estudios/2010/Top of mind 2010.pdf

[13] S.E. Kemp, T. Hollowood, J. Hort. Sensory Evaluation. A Practical Handbook. WileyBlackwell, 2009.

\section{E-mail: cminchonm@gmail.com}

